\title{
Identification of sas, a conserved gene cluster involved in the regulation of aerial mycelium formation in Streptomyces griseus
}

Correspondence

Kenji Ueda

ueda@brs.nihon-u.ac.jp

Received 24 June 2008

Revised 21 August 2008

Accepted 15 September 2008

\author{
Hideaki Takano, Takumi Tanaka, Teruhiko Beppu and Kenji Ueda
}

\author{
Life Science Research Center, College of Bioresource Sciences, Nihon University, 1866 Kameino, \\ Fujisawa 252-8510, Japan
}

\begin{abstract}
We cloned a DNA fragment that suppressed the aerial-mycelium-deficient phenotype in an amfS mutant of Streptomyces griseus when it was introduced into the cells via a high-copy-number plasmid. The sas $A B C D R$ gene cluster was identified as being responsible for this suppressive activity. The proteins encoded by sas $A B C D$ were of unknown function, but the operon structure was found to be conserved in all the strains of Streptomyces spp. and related organisms whose genomes have been sequenced. sas $R$, the flanking opposite coding sequence, encoded a putative DNA-binding protein. Subcloning revealed that the presence of all five coding sequences was essential for complete suppression. Scanning electron microscopy of Streptomyces griseus strains carrying the sas gene cluster at a high copy-number revealed that bundle-like structures consisting of several aerial hyphae were often formed. S1 nuclease protection analyses were performed to identify the transcriptional start site in the promoters preceding $\operatorname{sas} A$ and $\operatorname{sas} R$. The promoter preceding sas $A$ was highly active during vegetative growth. Null mutants for sas $A B C D$ among the $S$. griseus and $S$. coelicolor $A 3(2)$ cells exhibited bald phenotypes; this suggested a positive regulatory role of this gene cluster in the onset of morphogenesis in these two phylogenetically distinct Streptomyces species.
\end{abstract}

\section{INTRODUCTION}

Organisms of the Gram-positive bacterial genus Streptomyces are characterized by the ability to perform complex morphological differentiation in a manner similar to filamentous fungi. Early in their life cycle, these organisms develop branched multinucleoid hyphae that grow into the substrate (substrate mycelium). In response to nutrient limitations, the substrate mycelium produces aerial mycelium that culminates in long spore chains via the formation of septa at regular intervals (Chater, 1993). Extensive studies conducted on Streptomyces coelicolor A3(2), the model organism for genetic manipulation, have provided a deep understanding of cell development in Streptomyces spp.

We study the genetic regulation of the onset of aerial mycelium formation in Streptomyces griseus, the species that produces streptomycin. This organism is well characterized with regard to the role of the $\gamma$-butyrolactone autoregulatory factor called A-factor. S. Horinouchi and co-workers have precisely characterized the A-factor signalling cascade and identified multiple A-factor-dependent regulators as the master switches for the initiation of cell differentiation (Horinouchi, 2007).

In this study, we performed screening for a gene that suppresses the deficiency of aerial mycelium formation in an amfS mutant of $S$. griseus. It is assumed that AmfS, a morphogenic peptide equivalent to SapB/RamS of $S$. coelicolor A3(2), plays an important role in the erection of aerial hyphae under the control of A-factor (Ueda et al., 2002, 2005). Recently, the understanding of aerial mycelium formation in Streptomyces has greatly progressed based on the precise characterization of SapB in $S$. coelicolor A3(2) (reviewed by Willey et al., 2006). To date, however, no information is available with regard to suppressors of the phenotypic effects of a deficiency of this kind of morphogenic peptide. We anticipated that isolation of such suppressors will provide a new insight into morphogenesis in this group of bacteria. A DNA fragment responsible for the formation of aerial hyphae (albeit morphologically abnormal) in the amfS mutant was found to contain a conserved gene cluster of unknown function.

\section{METHODS}

Bacterial strains, plasmids and culture conditions. The wild-type strain of S. griseus (IFO13350) was obtained from the Institute of Fermentation, Osaka, Japan. Mutants for amfS (small coding sequence encoding a morphogenic peptide) and amfR (gene encoding a positive transcriptional regulator of amfTSBA operon) of S. griseus have been described previously (Ueda et al., 1998, 2002). The bld4 strain, a bald mutant of $S$. griseus, was obtained by performing 
$N$-methyl- $N^{\prime}$-nitro- $N$-nitrosoguanidine (NTG) mutagenesis in our laboratory. The genetic background of this mutant is not known; it lacks the ability to produce AmfS and forms abundant aerial hyphae upon the addition of exogenous AmfS peptide. A mutant for adpA (gene encoding the global transcriptional regulator for the onset of morphological and physiological development) (Ohnishi et al., 1999) was obtained from Y. Ohnishi (University of Tokyo, Japan). S. coelicolor A3(2) M130 was obtained from the John Innes Centre, Norwich, UK. Escherichia coli JM109 (Maniatis et al., 1982) was used as the host strain for DNA manipulation. pUC19 (Maniatis et al., 1982) was used for general DNA manipulation. TA cloning was performed for PCR-generated DNA fragments with the help of pT7Blue (Novagen). The enzymes used for DNA manipulation were purchased from Takara Shuzo. The culture conditions and genetic manipulation protocols used for E. coli and Streptomyces spp. were as described by Maniatis et al. (1982) and Kieser et al. (2000), respectively. Strains of Streptomyces spp. were grown in Bennett's/ sugar medium [containing $\left(\mathrm{g} \mathrm{l}^{-1}\right)$ : yeast extract (Difco), 1; meat extract (Kyokuto), 1; NZ amine (Wako Pure Chemical Industries), 2; and an appropriate sugar (Kokusan), 10 ( $\mathrm{pH} 7.2)]$, YMP/sugar medium [containing $\left(\mathrm{g} \mathrm{l}^{-1}\right)$ : yeast extract (Difco), 2; meat extract (Kyokuto), 2; Bacto-peptone (Difco), 4; $\mathrm{NaCl}, 5 ; \mathrm{MgSO}_{4} .7 \mathrm{H}_{2} \mathrm{O}, 2$; and an appropriate sugar (Kokusan), 10], ISP4 medium [containing $\left(\mathrm{g} \mathrm{l}^{-1}\right.$ ): soluble starch (Wako), $10 ; \mathrm{K}_{2} \mathrm{HPO}_{4}, 1 ; \mathrm{MgSO}_{4} .7 \mathrm{H}_{2} \mathrm{O}, 1 ; \mathrm{NaCl}$, 1; $\left(\mathrm{NH}_{4}\right)_{2} \mathrm{SO}_{4}, 2 ; \mathrm{CaCO}_{3}, 2$; and $1 \mathrm{ml} \mathrm{l}^{-1}$ trace salts solution containing $0.1 \mathrm{~g} \mathrm{l}^{-1}$ each of $\mathrm{FeSO}_{4} \cdot 4 \mathrm{H}_{2} \mathrm{O}, \mathrm{MnCl}_{2} \cdot 4 \mathrm{H}_{2} \mathrm{O}$ and $\left.\mathrm{ZnSO}_{4} \cdot 7 \mathrm{H}_{2} \mathrm{O}(\mathrm{pH} 7.2)\right]$ and $22 \mathrm{YE}$ medium (Kieser et al., 2000). Agar (1.5\%; Kokusan or Difco) was added to the above mixtures to obtain solid media. The plasmid pIJ702 (carrying genes responsible for thiostrepton resistance and melanin biosynthesis) (Katz et al., 1983 ) possesses a copy number of $40-400$ per genome, while the plasmid pXE4 has a low copy number of 1-2 per genome (Kieser et al., 2000). An integration plasmid, namely pKU464 carrying the phiBT1 integrase gene (Gregory et al., 2003), was obtained from $\mathrm{H}$. Ikeda (Kitasato University). For the selection of transformants from the E. coli cells, ampicillin (Wako) and neomycin (Wako) were used at a final concentration of $50 \mu \mathrm{g} \mathrm{ml}^{-1}$. For selecting the $S$. griseus transformants, thiostrepton (Sigma) and neomycin were used at a final concentration of $20 \mu \mathrm{g} \mathrm{ml}^{-1}$. A bioassay was performed for streptomycin according to a method described previously (Ueda et al., 2002).

Cloning of the sas gene cluster. The gene cluster described in this study was obtained by performing shotgun cloning using an amfS mutant as the host. BamHI-digested fragments of S. griseus genomic DNA were ligated to BglII-digested pIJ702 DNA and used to transform the host organism. A transformant, which grew as white colonies due to the formation of aerial mycelium, harboured a plasmid, pTT1, carrying a $7.2 \mathrm{~kb}$ fragment that contained coding sequences corresponding to SGR4456-4462 in the genome sequence database (http://www.genome.ad.jp/) (Ohnishi et al., 2008). The SGR4456 sequence in this plasmid lacked the $5^{\prime}$-terminal region corresponding to the four amino acids at the $\mathrm{N}$-terminus. This truncated coding sequence occurred as an in-frame fusion with melC1, a small coding sequence carried by pIJ702. The cloned fragment was then subcloned in the following manner. To construct pTT1-2, a DNA fragment that was amplified by PCR using the primers sasA-F and sasB-R (Table 1) was first TA cloned onto pT7Blue. Then, the fragment was excised as a BglII fragment and inserted at the BgIII site of pIJ702. Similar procedures were used to subclone the other plasmids. The inserts of pTT1-3, pTT1-4 and pTT1-5 were amplified by using sasA-F (Table 1) as the forward primer, and sasC-R, sasD-R and sasR-F, respectively (Table 1 ), as the reverse primers. The insert of pTT1-6 was amplified using sasR-R and sasR-F.
Electron microscopy. The hyphae of $S$. griseus and S. coelicolor $\mathrm{A} 3$ (2) strains that were grown at $28{ }^{\circ} \mathrm{C}$ for 6 days on ISP4 medium and YMP/glucose medium, respectively, were observed by scanning electron microscopy. To prepare the specimens, agar blocks were fixed with $2 \%$ osmium tetroxide for $30 \mathrm{~h}$ and were then dehydrated by freeze-drying. Each specimen was sputter-coated with palladium/ gold and examined using a VE8800 scanning electron microscope (Keyence).

S1 nuclease mapping. Transcription at the promoters preceding $s a s R$ and sasA was studied by S1 protection analysis. RNA was extracted from Streptomyces cells grown on cellophane-covered agar medium, and S1 nuclease mapping was performed, both using previously described methods (Kelemen et al., 2001; Kieser et al., 2000). A 285 bp hybridization probe was prepared by PCR using the primers sasR-F(S1) and sasR-R(S1) (Table 1) for both high- and lowresolution analysis of the $s a s R$ promoter. Similarly, the sasA promoter was analysed using a 278 bp hybridization probe prepared by PCR using the primers sasA-F and sasA-R(S1) (Table 1). All downstream primers were labelled at their $5^{\prime}$ end with $\left[\gamma^{-32} \mathrm{P}\right] \mathrm{ATP}$ using T4 polynucleotide kinase. The protected fragments were analysed on $6 \%$ polyacrylamide gels. A $100 \mathrm{bp}$ ladder marker (Takara) that was labelled with $\left[\gamma_{-}{ }^{32} \mathrm{P}\right] \mathrm{ATP}$ using T4 polynucleotide kinase was used as a standard to estimate the transcript sizes in the low-resolution assay. In the high-resolution assay, Maxam-Gilbert sequencing ladders $\left(\mathrm{A}+\mathrm{G}\right.$ and $\mathrm{C}+\mathrm{T}$ reactions) were generated using the ${ }^{32} \mathrm{P}$-labelled probe DNA fragment for the identification of the transcription start sites. Quality of RNA was assessed by performing a control assay for $h r d B$, which encodes a major sigma factor (Shinkawa et al., 1995).

Gene disruption. We generated sas $A B C D$-knockout mutants by a homologous recombination technique that was based on Redirect technology (Gust et al., 2003). The cosmid clone used for achieving knockout in S. griseus cells [containing the region corresponding to nucleotides 5202636-5245708 in the genome sequence database (http://www.genome.ad.jp/)] was available in our laboratory. SCF15, the cosmid used for achieving the knockout in S. coelicolor A3(2) cells, was obtained from the John Innes Centre. A cassette of an apramycinresistance gene, aac(3)IV, was prepared by PCR, using the primer sets DisG-F/DisG-R (for S. griseus) and DisC-F/DisC-R (for S. coelicolor) (Table 1). The sasABCD region in each cosmid clone was replaced with the apramycin-resistance gene cassette by performing in vivo recombination using the $\lambda$ Red system. The resulting apramycinresistant cosmid was then transformed into each organism. Subsequently, the apramycin-resistant recombinants were screened, and assessed for true recombination by performing PCR using appropriate primers. The integration plasmids used for genetic complementation of these knockout mutants were prepared in the following manner. The sas-coding region was amplified by PCR, using the primer sets sasA-F/sasD-R (for S. griseus) and sasA-F(SC)/sasD$\mathrm{R}(\mathrm{SC})$ (for S. coelicolor) (Table 1) and was cloned at the BamHI site of pKU464. The resultant plasmid was used to transform each organism to generate a kanamycin-resistant transformant harbouring the plasmid integrated at the BT1 site (Gregory et al., 2003). The mutant of sasR of S. griseus was obtained similarly as above, using primer sets DisGR-F/DisGR-R (Table 1).

\section{RESULTS}

\section{Cloning of the sas gene cluster}

We screened a library constructed in the high-copynumber plasmid pIJ702 for inserts that suppressed the aerial-mycelium-deficient phenotype of an amfS mutant of S. griseus, and obtained the plasmid pTT1 (see Methods). 
Table 1. Oligonucleotide primers used in this study

\begin{tabular}{|c|c|c|c|}
\hline Name & Sequence $\left(5^{\prime}-3^{\prime}\right)^{*}$ & Restriction enzyme & Position (nt) $\dagger$ \\
\hline sasA-F & AGATCTCAACAGGGCGGTGATGAC & $B g I I I$ & $5218636-5218655$ \\
\hline sasB-R & AGATCTACCATCAGCTGTCTCCTC & $B g I I I$ & $5217181-5217198$ \\
\hline sasC-R & AGATCTCTTCAGTGTCGTCTCGGTGTTC & $B g I I I$ & $5216553-5216574$ \\
\hline sasD-R & AGATCTCAGACCTCCAGGCCCGC & $B g I I I$ & $5216205-5216222$ \\
\hline sasR-R & AGATCTTCACTCCACGAAGAGAGCG & $B g I I$ & $5215931-5215949$ \\
\hline sasR-F & AGATCTTATCGATGTGCGCCAGACATG & $B g I I I$ & $5214823-5214843$ \\
\hline sasA-R(S1) & 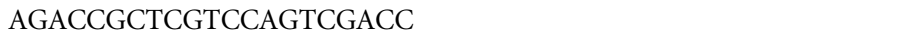 & - & $5218358-5218378$ \\
\hline sasR-F(S1) & TGCACTGCTGGCTGGGTCGCTC & - & $5214863-5214884$ \\
\hline sasR-R(S1) & TCGAAAAGCCGCTGCAACC & - & $5215129-5215147$ \\
\hline DisG-F & $\begin{array}{l}\text { TTACAGCCGTACGCCACGTCGGGAGGGGAGATCCGCGTGattccggggat- } \\
\text { ccgtcgacc }\end{array}$ & - & $5218441-5218479$ \\
\hline DisG-R & $\begin{array}{l}\text { TGCGGGGCCGGACCGAAGACCTCGCGGCGGCCGGGGTCAtgtaggctgg- } \\
\text { agctgcttc }\end{array}$ & - & $5216169-5216207$ \\
\hline DisGR-F & $\begin{array}{l}\text { GACCTTCCTGGCTGAATAGAACGGTCGGCGCCGGTCGTGattccggggat- } \\
\text { ccgtcgacc }\end{array}$ & - & $5215038-5215076$ \\
\hline DisGR-R & $\begin{array}{l}\text { GCGGCTGAGAATTCCGCGCGGGCCGGGAGCCCGCCCTCAtgtaggctgg- } \\
\text { agctgcttc }\end{array}$ & - & $5215985-5215947$ \\
\hline sasA-F(SC) & AGATCTGAGCTTCTTCACGTTGTCCAG & $B g I I I$ & $727333-727313$ \\
\hline sasD-R(SC) & AGATCTTCAGACCTCCAGTTCGCTCTC & $B g I I I$ & $724639-724659$ \\
\hline DisC-F & $\begin{array}{l}\text { GTTCGCGGTACAGCCGTGCAGGGGAGGGGATCCGGCGTGattccgggga- } \\
\text { tccgtcgacc }\end{array}$ & - & $726844-726806$ \\
\hline DisC- $\mathrm{R}$ & $\begin{array}{l}\text { GCGCCGGTCCCGCGGCGGGTGAACGGGCGGCCGGGATCAtgtaggctgg- } \\
\text { agctgcttc }\end{array}$ & - & $724603-724641$ \\
\hline
\end{tabular}

${ }^{\star}$ Sites for restriction are underlined. Sequences corresponding to the apramycin resistance gene are shown in lower case.

†Corresponding position in the genome sequence database of S. griseus (http://streptomyces.nih.go.jp/griseus/) and S. coelicolor A3(2) (http:// gib.genes.nig.ac.jp).

The amfS mutant cells harbouring pTT1 grew as white colonies due to the formation of aerial hyphae (Fig. 1a). Scanning electron microscopy of the white colonies demonstrated abundant growth of aerial mycelium with occasional unique bundle-like structures, reminiscent of fungal synnema structures, due to the adhesion of several hyphae (Fig. 1b). Occasional formation of spore chains was also seen (data not shown). The introduction of pTT1 into the cells of other bald mutants, including an amfR mutant (data not shown) and a bld4 mutant, as well as the wildtype strain (Fig. 1b), also resulted in the growth of unusual aerial mycelium. The introduction of pTT1 did not restore aerial mycelium formation in a knockout mutant of adpA, the central regulatory gene of the A-factor cascade that positively controls the transcription of A-factor-dependent regulatory genes including the amf cluster (Horinouchi, 2007) (data not shown). These results indicate that some adpA-dependent gene other than amf is essential for the suppressive effect of pTT1.

A cross-feeding assay revealed that the amfS mutant harbouring pTT1 did not induce the growth of aerial mycelium in an amfS mutant harbouring an empty plasmid growing in close proximity (Fig. 1c, left panel), while the wild-type strain did (Fig. 1c, right panel). This supported the view that the amfS mutants harbouring
pTT1 did not produce the AmfS peptide and that the gene cloned into pTT1 was not the amfS gene.

Subsequent nucleotide sequencing and search in the genome database revealed that pTT1 carried a $7.152 \mathrm{~kb}$ BamHI fragment at the BglII site of pIJ702. This region contained seven protein-coding sequences (CDSs) corresponding to SGR4456-SGR4462 (Fig. 2); SGR4456 was truncated at the $5^{\prime}$ terminus (see Methods). A subsequent subcloning experiment revealed that pTT1-4, containing SGR4457-4460, induced aerial mycelium formation at a slightly lower level in comparison to the original plasmid (Fig. 1a), and that pTT1-2 and pTT1-3, containing SGR4459-4460 and SGR4458-4460, respectively, did not (data not shown). The introduction of pTT1-5, carrying SGR4457-4460 along with the intact SGR4456, induced aerial mycelium formation to a high degree (Fig. 1a). The introduction of the intact SGR4456-4460 region into the cells via a low-copy-number plasmid (pXE4) did not induce the formation of aerial mycelium (data not shown). These results indicated that the introduction of four CDSs corresponding to SGR4457-4460 into the amfS mutant via a high-copy-number plasmid was essential to restore the formation of aerial mycelium and they raise the possibility that SGR4456 is also related to this restorative effect. On the basis of these observations, the five CDSs are hereafter 
(a)

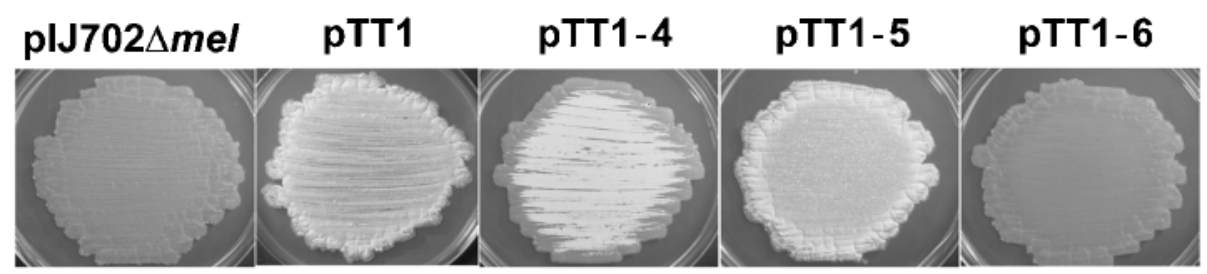

(b)
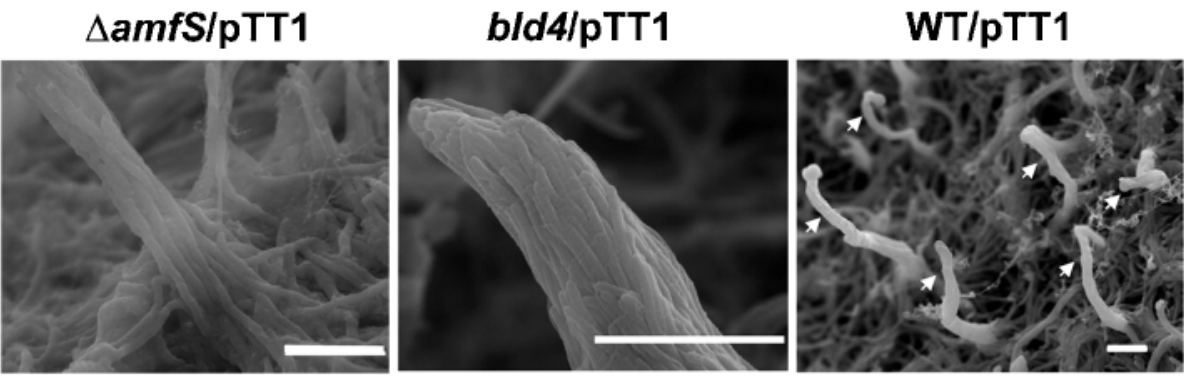

(c)

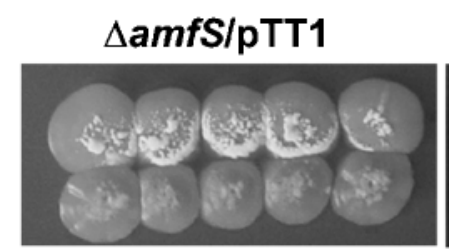

$\Delta$ amfS

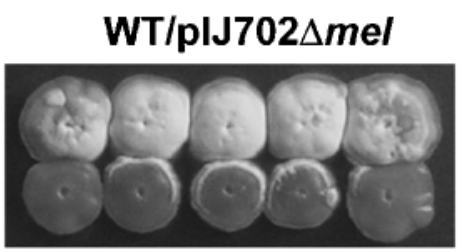

$\Delta a m f S$

Fig. 1. Phenotypes of $S$. griseus conferred by the introduction of the plasmid obtained in this study. (a) Colonies of the amfS mutant harbouring the original plasmid (pTT1) and its derivatives grown on YMP/glucose agar medium. The white colour of the colony is due to the development of aerial mycelium from the substrate mycelium, and the dark (brown) colour is due to the growth of only substrate mycelium. The region introduced into each plasmid is shown in Fig. 2. Patches were photographed on day 4. (b) Scanning electron micrographs of $S$. griseus strains (amfS mutant, b/d4 mutant and wild-type) harbouring pTT1. The introduction of the plasmid into the cells often caused the formation of bundle-like structures due to the adhesion of several aerial hyphae. Arrowheads indicate that the bundle-like structures also occurred in the wild-type carrying pTT1. Bars, 10 $\mu \mathrm{m}$. (c) A cross-feeding assay showing the secretion of AmfS. In the control assay (right panel), aerial mycelium was formed in the marginal region of the amfS mutant colonies (lower row) due to the activity of the AmfS peptide that was secreted by the wildtype colonies (upper row). In contrast, the amfS mutant cells that were grown in close proximity to the amfS mutant harbouring pTT1 (left panel) did not form aerial mycelium; this indicated that neither AmfS nor a substitute diffusible morphogen was produced by the amfS mutant harbouring PTT1.

designated sasABCDR (suppressor of aerial mycelium deficiency of amfS mutant).

The results of a database search (http://www.genome.ad.jp/) revealed that proteins encoded by $s a s A$ and $s a s D$ did not exhibit distinct homology with any proteins of known function. The SasA (137 aa) and SasD (124 aa) products were homologous to each other ( $35 \%$ identity). The results of a Pfam motif search revealed that the SasB product (269 aa) contained a helix-turn-helix domain in the Cterminal region. The major part $(24-113$ aa) of the SasC product (168 aa) exhibited a distinct similarity (E value $=7.6 \times 10^{-19}$ ) with the proteins of the roadblock/LC7 family. This family has been well characterized in terms of the association with eukaryotic dynein (Song et al., 2005), but it also includes a gliding motility protein $(\mathrm{Mgl})$ of Myxococcus xanthus (Stephens et al., 1989). The SasR product exhibited an end-to-end similarity with transcriptional regulators of the Rpi family (Sorensen \& Hove-Jensen, 1996). Currently no information is available with regard to the localization of Sas proteins, but none of them retained a distinct $\mathrm{N}$-terminal signal sequence for secretion. 
(a)

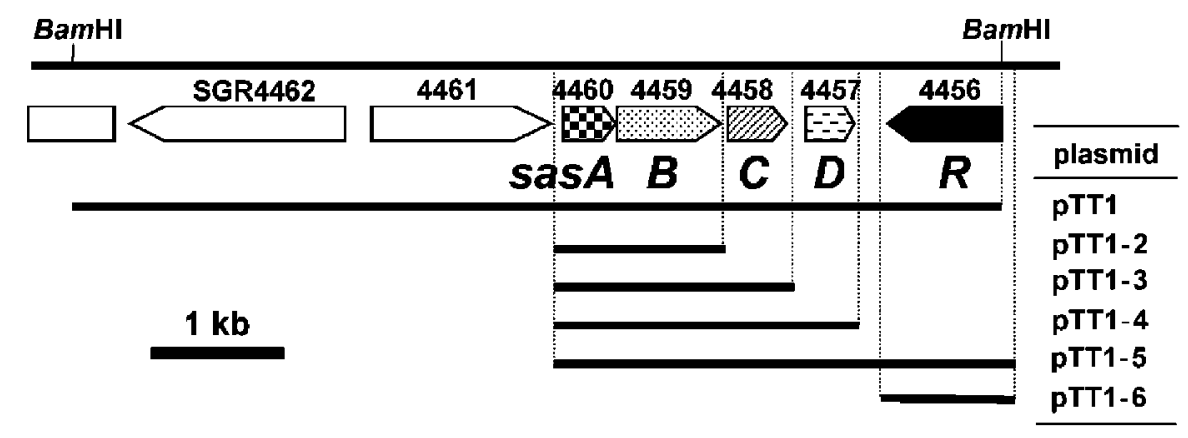

(b)

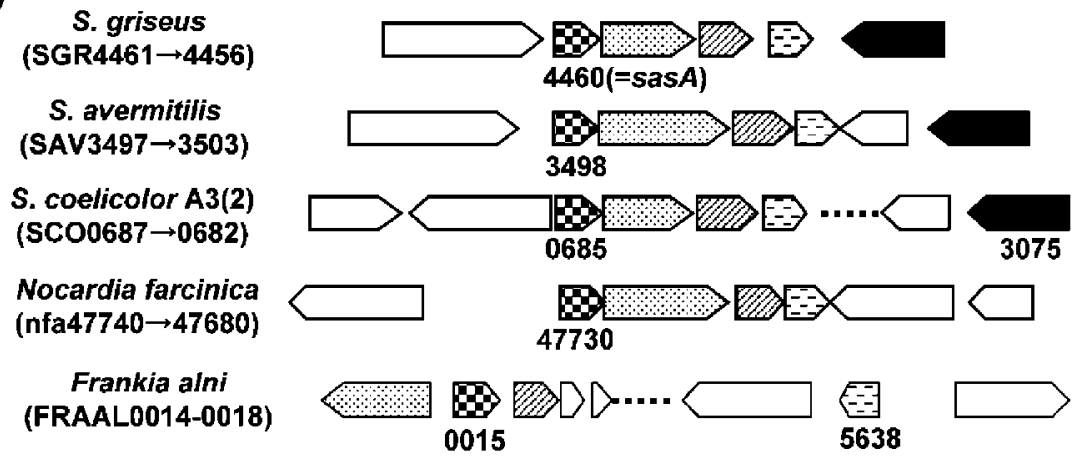

Fig. 2. Schematic representation of the gene cluster carried by pTT1. (a) The BamHI region of $S$. griseus that was cloned onto pTT1. The region contained seven complete coding sequences, including sas $A B C D R$, but it lacked the $5^{\prime}$ region of sas $R$. In this construction, a plasmid-borne promoter involved in melanin biosynthesis (Katz et al., 1983) directed the expression of sas $R$, which was in-frame with a small coding sequence (me/C1) on this plasmid. The regions corresponding to the subcloned fragment are also shown. SGR4461 and 4462 encode a putative amino acid permease and urocanate hydratase, respectively. pTT1-5 and pTT1-6 contain the intact promoter region preceding sas $R$. (b) The sas gene cluster homologues. The sas $A B C D$ gene cluster was found to be conserved in all Streptomyces spp. whose complete genome sequences are known. The cluster was also found in the genome of $N$. farcinica. The sas $R$ homologue was located in the same locus as sas $A B C D$ in $S$. avermitilis but in a different locus in S. coelicolor A3(2). The sasR homologue was not found in N. farcinica and F. alni.

The sasABCD cluster was also found in the genomes of Streptomyces avermitilis, S. coelicolor A3(2) and Nocardia farcinica. The short length of the intergenic regions $(-10$ to $54 \mathrm{bp})$ supported the view that $\operatorname{sas} A B C D$ constituted an operon structure in these organisms. This also suggested that the four proteins in this operon are functionally related and that they play a general role in this group of bacteria. The sas $A B C D$ gene cluster homologue was not found in the genomes of other organisms, although homologues of each component were present in the genomes of various bacteria, including cyanobacteria and Gram-negative bacteria such as Myxococcus and Xanthomonas. The sasR homologue of $S$. avermitilis was located in the flanking region of the sas $A B C D$ cluster, while that of S. coelicolor A3(2) was located at a different locus. The sas $R$ homologue was not present in the genome of $N$. farcinica. sas $A B C$ comprised a cluster in Frankia alni, but the sasD homologue was in a different locus (Fig. 2b).

\section{Transcription of the sas gene cluster}

The transcriptional activity of the promoters preceding $s a s A$ and sasR was studied by performing S1 nuclease protection analyses. A high-resolution analysis assigned the unique transcriptional start site in both the promoters (Fig. $3 a, b)$. Potential -35 and -10 sequences were found in the sasR promoter (TGGACA...CTGAAT), but not in the sasA promoter.

The low-resolution S1 analysis revealed that the activity of the sas A promoter was relatively high and especially active during the vegetative growth phase (Fig. 3c). On the other hand, the activity of the sasR promoter was very low and was observed during the early growth phase (data not shown). These two promoters exhibited similar transcriptional activity in a disruption mutant of $\operatorname{adp} A$, the central regulator of the A-factor cascade (Ohnishi et al., 1999) (data not shown). 


\section{(a)}

CAACAGGGCGGTGATGACGGCCCGCGAGAAGTCGGGCGCCCCGGGCGCCTGACCGGACGG GGCCGACGCATCCGCCACCGCCTTTCCGGCCGCTCCTTTACACCGCGGGCCGCATGTACC AAGGGAAAATGCCAGAACCCCCACCGGCCGTGAGCACGATGCGTAGCCTCAGCGTTACAG WhunwCCGTACGCCACGTCGGGAGGGGAGATCCGCGTGCCCGGAATCGACGAGTGCCTGCTCGA.

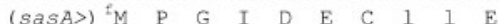

CACTGCTGGCTGGGTGGCTCCCGGTCAGCACGAGCGCGTGCGGCTAGACGACTGGCGCGG CTACGCGCAAACCCGACTGCGGTCAGGTTTCTCCGCCTGTTGGGCTTCGGCTCTCGAACC GGTACGGCCCTTCTCGCACTGCCCCGGCCTACCCCCTGCATATGGACATACAGCGACCTT $+1$

CCTGGCTGAATAGAACGGTCGGCGCCGGTCGTGGCACGACGGATCCAGCAAGGGGACAGC

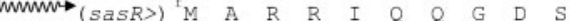

(b)
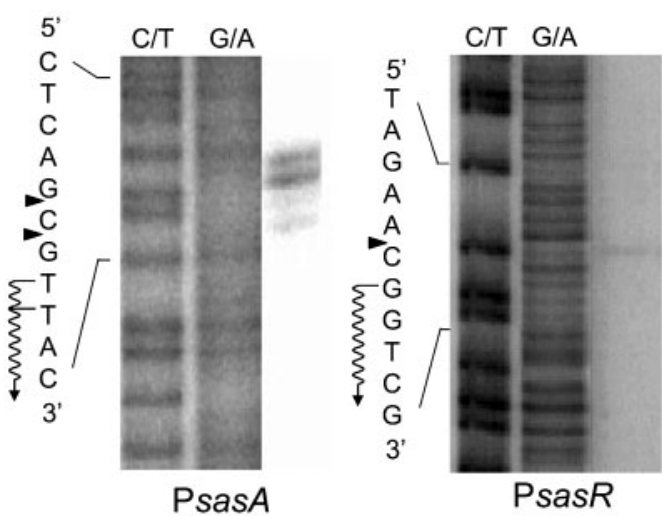

(c)

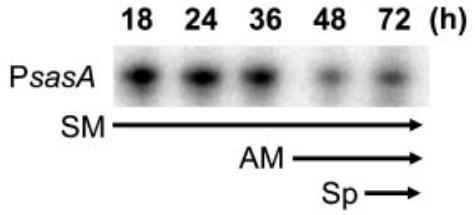

\section{Phenotype of knockout mutants}

To study the role of the sas gene cluster, null mutants with deletions of the four coding sequences $(\triangle \operatorname{sas} A B C D)$ were generated in $S$. griseus and $S$. coelicolor A3(2) (see Methods). The sas $A B C D$ mutant of $S$. griseus was defective in the formation of aerial mycelium (bald phenotype) on ISP4 agar (Fig. 4). This mutant phenotype was dependent on the medium composition; the mutant exhibited the wild-type morphotype when cultured on media such as Bennett's/glucose and YMP/glucose. The level of streptomycin produced by the mutant was the same as that produced by the wild-type strain in various media with different compositions (data not shown). We also obtained a null mutant of sasR of $S$. griseus, and found that it exhibited a wild-type phenotype on all media tested (data not shown).

The sasABCD mutant of $S$. coelicolor A3(2) also exhibited a bald phenotype (Fig. 5). When cultured on YMP/glucose agar medium, the mutant did not form aerial mycelium but produced the same levels of actinorhodin (the blue
Fig. 3. $\mathrm{S} 1$ nuclease protection analyses of the promoters preceding $\operatorname{sas} A$ and $\operatorname{sas} R$. (a) Nucleotide sequence of the promoter region preceding sas $A$ (upper block) and sas $R$ (lower block). The transcriptional start positions determined by highresolution analyses are indicated by the wavy arrows. The possible -35 and -10 regions of sas $R$ promoter are underlined. (b) Highresolution analyses for the determination of transcriptional start sites of the promoters preceding sas $A$ and sas $R$. Maxam-Gilbert sequencing ladders $(C+T$ and $G+A$ reactions) were generated with the same ${ }^{32} \mathrm{P}$ fragment as the probe DNA. The positions of the S1-protected fragment are shown by arrowheads and the transcriptional start sites were assigned to the residues indicated by the wavy arrows. It is known that the fragments generated by the chemical sequencing reactions migrate 1.5 nucleotides further than the corresponding fragments generated by $\mathrm{S} 1$ nuclease digestion of the DNA-RNA hybrids (half a residue from the presence of the $3^{\prime}$-terminal phosphate group and one residue from the elimination of the $3^{\prime}$-terminal nucleotide) (Sollner-Webb \& Reeder, 1979). The RNA prepared from the wild-type cells grown for 3 days on YMP solid medium containing glucose was used for hybridization. (c) Low-resolution S1 analysis. The activity of the sas $A$ promoter was estimated by the intensity of the hybridization signal. RNA was extracted from the cells of each $S$. griseus strain, grown on YMP solid medium containing $1 \%$ glucose for the indicated periods. The wild-type strain grew as substrate mycelium (SM) at day 1 , as a mixture of substrate and aerial mycelium (AM) at day 2 and as a mixture of aerial mycelium and spores (Sp) at day 3. Quality of RNA was confirmed by a control assay for $h r d B$ (data not shown).

diffusible pigment antibiotic) as did the wild-type cells. The mutant was also defective in the formation of aerial mycelium when cultured on $\mathrm{YMP} /$ maltose and R2YE media. Aerial growth was restored in the sas $A B C D$ mutants of $S$. griseus and S. coelicolor by transforming them with an integration vector containing the intact gene cluster (data not shown).

\section{DISCUSSION}

In this study, we identified sas as a new locus involved in morphological development in S. griseus. The wide distribution of this gene cluster suggests that it plays a general role in Streptomyces spp. and related bacteria. The information currently available regarding the function of homologous proteins is insufficient for deducing the function of the Sas products. Although our evidence supported the view that Sas proteins take part in a particular process, we could not detect distinct interactions among Sas proteins in a two-hybrid-system analysis that was performed using E. coli as a host (our unpublished observation). Hence, we arrived at the assumption that the proteins do not form a complex with strong associations.

The activation of promoters preceding the sas genes during the early growth phase suggests that the role of Sas proteins is specific to vegetative cells. The fact that these promoters exhibited wild-type activities in the 


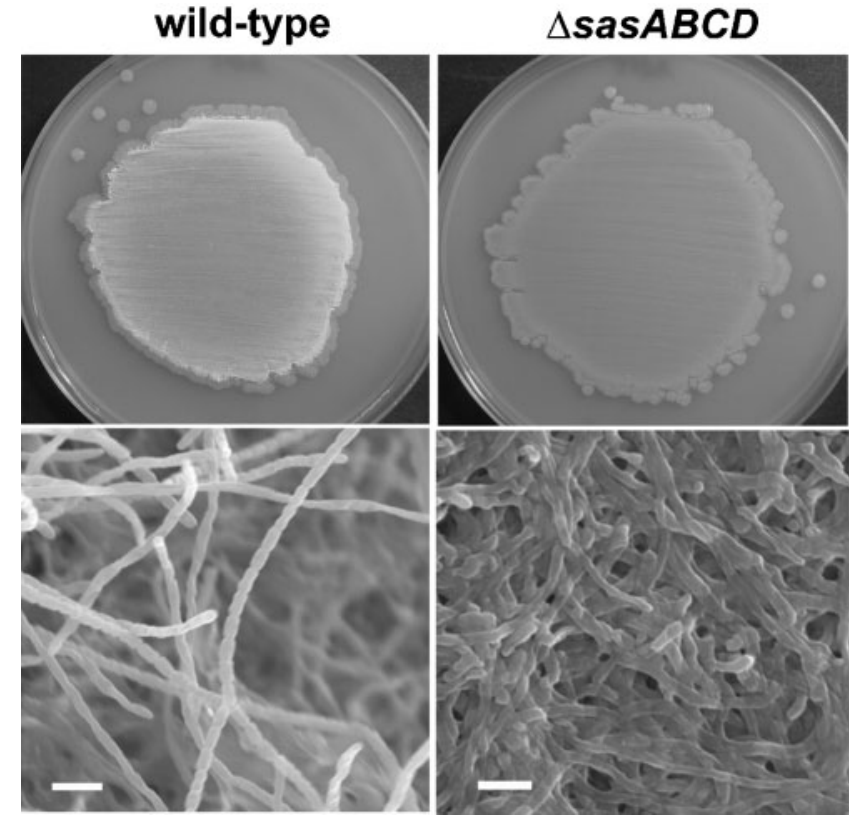

Fig. 4. Macroscopic and microscopic observation of solid culture of an sas $A B C D$ mutant of $S$. griseus. The parental and sas $A B C D$ mutant strains were grown on ISP 4 solid medium for 4 days. Bars, $5 \mu \mathrm{m}$.

adpA mutant indicates that the expression of sas genes is independent of the A-factor cascade. The result of subcloning (Figs 1a and 2a) raises the possibility that the SasR product, a putative transcriptional regulator, controls the activity of the promoter preceding sasA, but we have not yet confirmed the binding of SasR protein to the promoter region. The fact that knockout of sasR did not affect the wild-type phenotype in $S$. griseus supports the view that the role of $s a s R$ is not significantly related to that of $\operatorname{sas} A B C D$, although the close location of the coding sequences still implies some functional relationship. Characterization of the transcriptional control of the sas gene cluster may provide new information with regard to developmental signalling in Streptomyces.

The introduction of the sas gene cluster into amfS mutant cells via a high-copy-number plasmid induced aerial mycelium formation. We previously found that amfS encodes morphogenic peptides essential for the formation of aerial mycelium in S. griseus (Ueda et al., 2002). Evidence from a recent study on S. coelicolor A3(2) has indicated that AmfS produced by $S$. griseus is a homologue of the morphogenic hydrophobic peptide SapB in S. coelicolor A3(2) (Willey et al., 2006). Hence, AmfS is currently assumed to be a hydrophobin that induces the formation of aerial mycelium by reducing the surface tension of the substrate mycelium, based on the knowledge gained from the study of S. coelicolor A3(2).

A possible explanation for the suppressive effect of sas is that its overexpression induced the production of a hydrophobic morphogen alternative to AmfS. However, the results of a

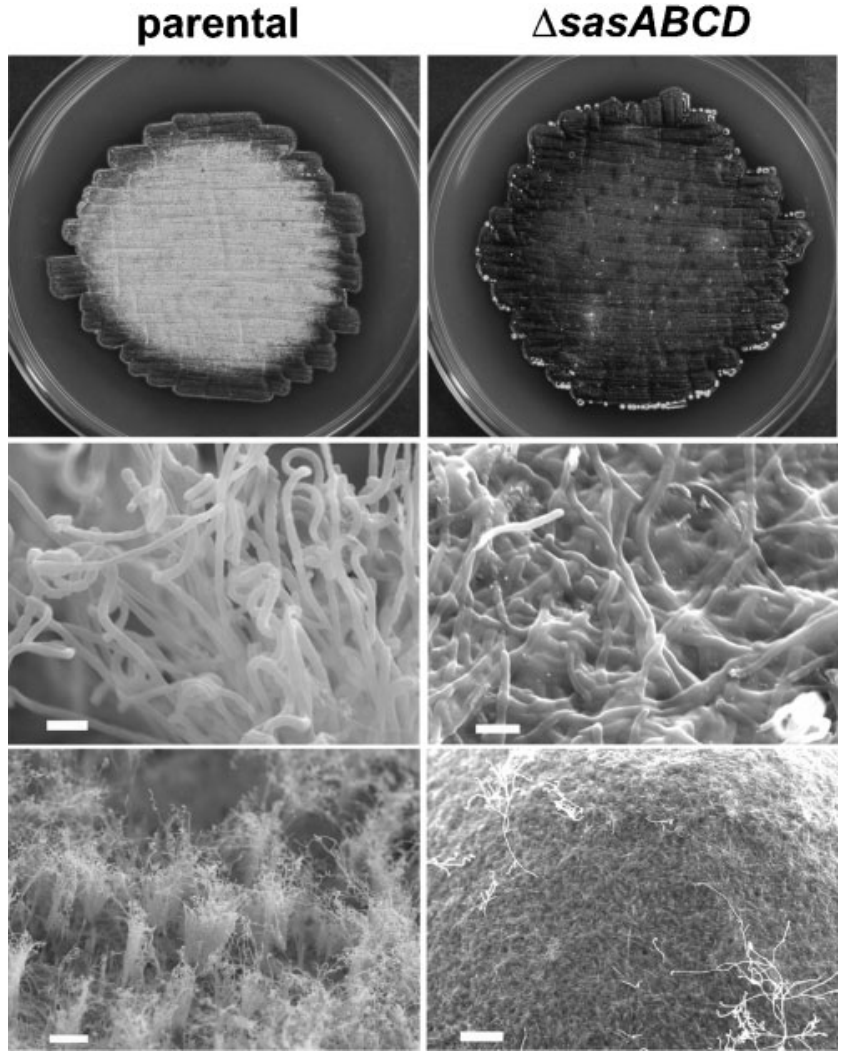

Fig. 5. Macroscopic and microscopic observation of solid culture of an sas $A B C D$ mutant of $S$. coelicolor $A 3(2)$. The parental and sas $A B C D$ mutant strains were grown on YMP solid medium containing $1 \%$ glucose for 4 days. Bars, $2 \mu \mathrm{m}$ (upper) and $20 \mu \mathrm{m}$ (lower).

cross-feeding assay (Fig. 1c) did not reveal the secretion of any diffusible morphogenic substance by the amfS mutant harbouring pTT1. Another possibility is that the sas genes are associated with the cellular responses to hydrophobins. Although the existence of such a mechanism has not yet been confirmed, the possibility that some specific system is involved in or associated with the response of substrate mycelium to exogenous hydrophobins could prove to be a persuasive argument. The marked adhesion of aerial mycelium that was induced by the introduction of sas genes in various strains (Fig. 1b) leads to the assumption that it has some relationship with the mechanism of cell-surface activation. Further investigations on the functions of the Sas proteins may reveal other aspects of the complex morphological development in Streptomyces spp.

\section{ACKNOWLEDGEMENTS}

We thank Y. Ohnishi and S. Horinouchi for providing the adpA mutant, and $\mathrm{H}$. Ikeda for providing the integration plasmid. This study was supported by the High-Tech Research Center Project of the Ministry of Education, Culture, Sports, Science and Technology, Japan. 


\section{REFERENCES}

Chater, K. F. (1993). Genetics of differentiation in Streptomyces. Annu Rev Microbiol 47, 685-713.

Gregory, M. A., Till, R. \& Smith, M. C. (2003). Integration site for Streptomyces phage phiBT1 and development of site-specific integrating vectors. J Bacteriol 185, 5320-5323.

Gust, B., Challis, G. L., Fowler, K., Kieser, T. \& Chater, K. F. (2003). PCR-targeted Streptomyces gene replacement identifies a protein domain needed for biosynthesis of the sesquiterpene soil odor geosmin. Proc Natl Acad Sci U S A 100, 1541-1546.

Horinouchi, S. (2007). Mining and polishing of the treasure trove in the bacterial genus Streptomyces. Biosci Biotechnol Biochem 71, 283-299.

Katz, E., Thompson, C. J. \& Hopwood, D. A. (1983). Cloning and expression of the tyrosinase gene from Streptomyces antibioticus in Streptomyces lividans. J Gen Microbiol 129, 2703-2714.

Kelemen, G. H., Viollier, P. H., Tenor, J., Marri, L., Buttner, M. J. \& Thompson, C. J. (2001). A connection between stress and development in the multicellular prokaryote Streptomyces coelicolor A3(2). Mol Microbiol 40, 804-814.

Kieser, T., Bibb, M. J., Buttner, M. J., Chater, K. F. \& Hopwood, D. A. (2000). Practical Streptomyces Genetics. Norwich, UK: John Innes Foundation.

Maniatis, T., Fritsch, E. F. \& Sambrook, J. (1982). Molecular Cloning: a Laboratory Manual. Cold Spring Harbor, NY: Cold Spring Harbor Laboratory Press.

Ohnishi, Y., Kameyama, S., Onaka, H. \& Horinouchi, S. (1999). The A-factor regulatory cascade leading to streptomycin biosynthesis in Streptomyces griseus: identification of a target gene of the A-factor receptor. Mol Microbiol 34, 102-111.

Ohnishi, Y., Ishikawa, J., Hara, H., Suzuki, H., Ikenoya, M., Ikeda, H., Yamashita, A., Hattori, M. \& Horinouchi, S. (2008). Genome sequence of the streptomycin-producing microorganism Streptomyces griseus IFO 13350. J Bacteriol 190, 4050-4060.

Shinkawa, H., Hatada, Y., Okada, M., Kinashi, H. \& Nimi, O. (1995). Nucleotide sequence of a principal sigma factor gene $(h r d B)$ of Streptomyces griseus. J Biochem 118, 494-499.

Sollner-Webb, B. \& Reeder, R. H. (1979). The nucleotide sequence of the initiation and termination sites for ribosomal RNA transcription in X. laevis. Cell 18, 485-499.

Song, J., Tyler, R. C., Lee, M. S., Tyler, E. M. \& Markley, J. L. (2005). Solution structure of isoform 1 of Roadblock/LC7, a light chain in the dynein complex. J Mol Biol 354, 1043-1051.

Sorensen, K. I. \& Hove-Jensen, B. (1996). Ribose catabolism of Escherichia coli: characterization of the $r p i B$ gene encoding ribose phosphate isomerase $\mathrm{B}$ and of the $r p i R$ gene, which is involved in regulation of rpiB expression. J Bacteriol 178, 1003-1011.

Stephens, K., Hartzell, P. \& Kaiser, D. (1989). Gliding motility in Myxococcus xanthus: $m g l$ locus, RNA, and predicted protein products. J Bacteriol 171, 819-830.

Ueda, K., Hsheh, C. W., Tosaki, T., Shinkawa, H., Beppu, T. \& Horinouchi, S. (1998). Characterization of an A-factor-responsive repressor for $a m f R$ essential for onset of aerial mycelium formation in Streptomyces griseus. J Bacteriol 180, 5085-5093.

Ueda, K., Oinuma, K., Ikeda, G., Hosono, K., Ohnishi, Y., Horinouchi, S. \& Beppu, T. (2002). AmfS, an extracellular peptidic morphogen in Streptomyces griseus. J Bacteriol 184, 1488-1492.

Ueda, K., Takano, H., Nishimoto, M., Inaba, H. \& Beppu, T. (2005). Dual transcriptional control of amfTSBA, which regulates the onset of cellular differentiation in Streptomyces griseus. J Bacteriol 187, 135-142.

Willey, J. M., Willems, A., Kodani, S. \& Nodwell, J. R. (2006). Morphogenetic surfactants and their role in the formation of aerial hyphae in Streptomyces coelicolor. Mol Microbiol 59, 731-742.

Edited by: M. Paget 